\title{
Disturbance Compensating Model Predictive Control With Application to Ship Heading Control
}

\author{
Zhen Li, Member, IEEE, and Jing Sun, Fellow, IEEE
}

\begin{abstract}
To address the constraint violation and feasibility issues of model predictive control (MPC) for ship heading control in wave fields, a novel disturbance compensating MPC (DC-MPC) algorithm has been proposed to satisfy the state constraints in the presence of environmental disturbances. The capability of the novel DC-MPC algorithm is first analyzed. Then, the proposed DC-MPC algorithm is applied to solve the ship heading control problem, and its performance is compared with a modified MPC controller, which considers the estimated disturbance in the optimization directly. The simulation results show good performance of the proposed controller in terms of reducing heading error and satisfying yaw velocity and actuator saturation constraints. The DC-MPC algorithm has the potential to be applied to other motion control problems with environmental disturbances, such as flight, automobile, and robotics control.
\end{abstract}

Index Terms-Disturbance estimation, model predictive control (MPC), ship heading control, state constraints.

\section{INTRODUCTION}

S HIP heading control, also known as course keeping, has been a representative control problem for marine applications and has attracted considerable attention from the control community (see [1] and [2], and references therein). The traditional ship heading controllers did not consider the inherent physical limitations in the control inputs, namely, the rudder saturation. More recently, given that the large yaw velocity can produce other motions (such as sway and roll) that can cause seasickness and cargo damage, enforcing yaw velocity constraints while maneuvering in seaways becomes an important design consideration in surface vessel control. While typical nonlinear control methodologies do not take these input and output constraints explicitly into account in the design process, the constraint enforcement is often achieved through numerical simulations and trial-and-error tuning of the controller parameters. Few other control methodologies, such as the model predictive control (MPC) [3], [4] and reference governor [5], have a clear advantage in addressing input and state constraints explicitly. In [6], rudder saturation in the MPC controller for tracking control of marine surface vessels is considered, and in [7], the roll reduction for the heading control problem using an MPC approach is achieved. However, no state constraints, such as yaw rate and roll angle, are explicitly considered in [7]. The path following

Manuscript received August 17, 2010; accepted December 15, 2010. Manuscript received in final form January 09, 2011. Date of publication February 17, 2011; date of current version December 14, 2011. Recommended by Associate Editor R. Rajamani.

The authors are with the Department of Naval Architecture and Marine Engineering, University of Michigan, Ann Arbor, MI 48109 USA (e-mail: lizhen@umich.edu).

Color versions of one or more of the figures in this paper are available online at http://ieeexplore.ieee.org.

Digital Object Identifier 10.1109/TCST.2011.2106212 with input (rudder) and state (roll) constraints is achieved via $\mathrm{MPC}$ in [8].

MPC is a control technique, which embeds optimization within feedback to deal with systems subject to constraints on inputs and states [3], [4]. Using an explicit model, and the current measured or estimated state as the initial state to predict the future response of a plant, MPC determines the control action by solving a finite-horizon open-loop optimal control problem online at each sampling interval. Furthermore, because of its natural appeal to multi-variable systems, MPC can handle under-actuated or over-actuated problem gracefully by combining all the objectives into a single objective function.

One of the primary reasons for the success of MPC in industrial applications is its capability in enforcing various types of constraints on the process [9]. However, it may happen at some time steps that the constrained optimization problem the MPC is attempting to solve becomes infeasible due to the presence of model mismatches and/or disturbances. Namely, no solution can be found that satisfies all constraints. As an example, wave disturbances may cause infeasibility of standard MPC ship heading controller.

To address the feasibility issues in MPC applications in the presence of disturbance and model uncertainties, such as ship heading control in wave fields, numerous studies on robust MPC have been pursued, and the efforts have led to extensive publications in the literature ([3], [4], and [10], and references therein). The typical robust MPC approaches often consider bounded disturbances [11]-[15], assuming that they are confined to a compact set and allowed to take values within the set. However, this assumption ignores the knowledge of the disturbance dynamics and may lead to conservative results when such knowledge is available. Furthermore, these robust MPC algorithms are normally computationally intensive. Aiming to reduce the conservativeness in and to reduce conservativeness. Inspired by [16], this paper proposes a computationally efficient two-step algorithm to handle disturbance by exploring the disturbance information.

The contributions of this study lie in the following aspects.

1) A novel two-step disturbance compensating MPC (DCMPC) algorithm is proposed to achieve state constraint satisfaction and successive feasibility for linear systems with environmental disturbances. The theoretical proof is also provided.

2) Compared to the standard MPC, the proposed MPC controller is designed to achieve good system performance with low additional computational effort by leveraging "measurable" disturbances.

3) The proposed DC-MPC algorithm is successfully applied to ship heading control in wave fields to satisfy the yaw velocity constraints. 
This paper is organized as follows. The problem statement is given in Section II, and the DC-MPC algorithm is developed in Section III. In Section IV, the DC-MPC algorithm is applied to ship heading control with yaw velocity constraints, followed by the conclusions in Section V.

\section{PRoBlem STATEMENT}

Consider a discrete-time linear time-invariant system with disturbances

$$
x(k+1)=A x(k)+B u(k)+w(k), \quad w \in W
$$

where $x \in \mathbb{R}^{n_{o}}$ is the system state, $u \in \mathbb{R}^{n_{i}}$ is the control, and $w \in \mathbb{R}^{n_{o}}$ is an unknown disturbance taking values in the set $W$. $A$ and $B$ are system matrices assumed to be known.

The standard MPC considers the following optimization problem $\mathcal{P}^{\prime}(x(k))$ :

$$
\begin{aligned}
& \min _{u(\cdot \mid k)} \sum_{j=1}^{N_{p}}\left[x(k+j \mid k)^{T} Q x(k+j \mid k)\right. \\
& \left.\quad+u(k+j-1 \mid k)^{T} R u(k+j-1 \mid k)\right]
\end{aligned}
$$

subject to

$$
\begin{aligned}
& x(k+j+1 \mid k)=A x(k+j \mid k)+B u(k+j \mid k) \\
& x(k \mid k)=x(k) \\
& C x(k+j+1 \mid k) \leq D \\
& S u(k+j \mid k) \leq T
\end{aligned}
$$

where $j=0,1, \ldots, N_{p}-1,(3)$ is the nominal system dynamic equation used to predict the future states, and (5) and (6) are general state and input constraints, respectively, which are defined by matrices $C, D, S$, and $T$; the inequalities (5) and (6) are satisfied element by element. $Q$ and $R$ are the corresponding weighting matrices that are used to shape the responses, $N_{p}$ is the predictive horizon, $x(k+j \mid k)$ and $u(k+j \mid k)$ are the state and control, respectively, $j$ steps ahead of the current time $k$, and $x(k)$ is the (measured or estimated) state at time $k$.

If the optimization problem $\mathcal{P}^{\prime}(x(k))$ is feasible, then its corresponding optimal solution is denoted by $\left\{u^{\prime \prime *}(k \mid k), u^{\prime \prime *}(k+\right.$ $\left.1 \mid k), \ldots, u^{\prime \prime *}\left(k+N_{p}-1 \mid k\right)\right\}$. Accordingly, the predicted optimal states are $\left\{x^{\prime \prime *}(k+1 \mid k), x^{\prime \prime *}(k+2 \mid k), \ldots, x^{\prime \prime *}(k+\right.$ $\left.\left.N_{p} \mid k\right)\right\}$. For the standard MPC approach, the control action for the system (1) is chosen to be the first vector in the optimal sequence, i.e.,

$$
u(k)=u^{\prime \prime *}(k \mid k) .
$$

With disturbances $(w \neq 0)$, even if the optimization problem $\mathcal{P}^{\prime}(x(k))$ is feasible at time step $k$, the feasibility of the MPC optimization problem cannot be guaranteed at the next step $k+$ 1. More specifically, $C x(k+1) \leq D$ cannot be guaranteed with the solution given by (7).

One goal of this paper is to ensure repeated feasibility of problem $\mathcal{P}^{\prime}(x(k))$. Namely, if $C x(k) \leq D$ is satisfied, we want to guarantee that $C x(k+1) \leq D$ can be satisfied. The other goal of the design is to make the response of the system in the presence of disturbances as close as possible to the response without disturbances, assuming that the performance without disturbances reflects the design target when the MPC controller is properly implemented. The latter design objective can be expressed as to force $x(k+1) \rightarrow x^{\prime \prime *}(k+1 \mid k)$ in the presence of disturbance, which is not considered in the standard MPC design.

\section{Disturbance COMPENSATING MPC}

The disturbances at time step $k-1$, i.e., $w(k-1)$, can be estimated by the following equation if the state and control are measurable [16]:

$$
\hat{w}(k-1)=x(k)-A x(k-1)-B u(k-1) .
$$

When the sampling time $T_{s}$ is small and/or the disturbance changes slowly with time, we can make the following assumption.

Assumption 1: The disturbance at time step $k$, i.e., $w(k)$, can be estimated by

$$
w(k)=\hat{w}(k-1)+\varepsilon
$$

where $\varepsilon \in V$ and $V \subset W$. Please note that $\varepsilon$ can also capture small model mismatch other than the disturbance variation between time step $k$ and $k-1$.

Remark 1: If the sampling rate is very fast compared with the disturbance changing rate, the disturbance variate $\varepsilon$ will be very small, and the bound on $V$ will be much tighter than that for $W$. One important consideration in selecting the sampling rate of the MPC is the available computation recourses. Assumption 1 is valid for applications, where computational resource is not an issue and fast sampling can be implemented. For the application studied in this paper, namely, ship heading control, the sampling time can be chosen as fast as $0.5 \mathrm{~s}$ given a normal computer ( $2 \mathrm{GHz}$ CPU and $1024 \mathrm{MB}$ RAM) with a satisfactory 80 step prediction horizon. Compared with the dominant period of normal ocean waves (about 6-10 s), Assumption 1 holds for ship heading control in wave fields.

With Assumption 1, a straightforward approach would be to utilize the disturbance information directly in the optimization problem. Specifically, the following optimization problem $\mathcal{P}^{\prime \prime}(x(k), \hat{w}(k-1))$ is proposed by:

$$
\begin{aligned}
& \min _{u(\cdot \mid k)} \sum_{j=1}^{N_{p}}\left[x(k+j \mid k)^{T} Q x(k+j \mid k)\right. \\
& \left.\quad+u(k+j-1 \mid k)^{T} R u(k+j-1 \mid k)\right]
\end{aligned}
$$

subject to

$$
\begin{aligned}
& x(k \mid k)=x(k) \\
& x(k+1 \mid k)=A x(k \mid k)+B u(k \mid k)+\hat{w}(k) \\
& x(k+j+1 \mid k)=A x(k+j \mid k)+B u(k+j \mid k), \\
& \quad j=1, \ldots, N_{p}-1 \\
& C x(k+j+1 \mid k) \leq D, \quad j=0,1, \ldots, N_{p}-1 \\
& S u(k+j \mid k) \leq T, \quad j=0,1, \ldots, N_{p}-1 .
\end{aligned}
$$

The first element of the optimal sequence of $\mathcal{P}^{\prime \prime}(x(k), \hat{w}(k-1))$ is implemented as the control input to the system (1). This MPC 
scheme modifies the linear model by considering the next step disturbance to predict the future states; thus, it will be refereed as modified MPC (M-MPC) in the sequel.

Using the M-MPC scheme, the state constraints normally can be satisfied. However, the system performance is not satisfactory for the ship heading control. One can refer to the simulation results summarized in Section IV. To improve the system responses, the DC-MPC scheme is proposed to not only satisfy the state constraints in the presence of disturbance, but also to retain the performance level achieved by the system in calm water (without disturbance). The design of the DC-MPC involves several steps as described in the sequel.

Step 1: At time step $k$, calculate the disturbance $\hat{w}(k-1)$ of the previous time step $k-1$ using (8), and the measured values of $x(k), x(k-1)$, and $u(k-1)$.

Step 2: Calculate the disturbance compensation control $\Delta u$ by solving the following low-dimension optimization problem $\mathcal{P}_{\Delta}(\hat{w}(k-1))$ :

subject to

$$
\min _{\Delta u \in \mathbb{R}^{n_{i}}}\|C B \Delta u+C \hat{w}(k-1)\|
$$

$$
\begin{aligned}
& C B \Delta u \leq-C \hat{w}(k-1)-E \\
& S \Delta u \leq T
\end{aligned}
$$

where $E=\max (C \varepsilon)$ with $\varepsilon \in V$. Suppose the corresponding optimal solution for $\mathcal{P}_{\Delta}(\hat{w}(k-1))$ is $\Delta u^{*}$. In a special case when $\hat{w}(k-1)=0$, we have $\Delta u^{*}=0$, which leads to the standard MPC.

Step 3: Solve the optimization problem $\mathcal{P}\left(x(k), \Delta u^{*}\right)$ as follows:

$$
\begin{aligned}
& \min _{u(\cdot \mid k)} \sum_{j=1}^{N_{p}}\left[x(k+j \mid k)^{T} Q x(k+j \mid k)\right. \\
& \left.\quad+u(k+j-1 \mid k)^{T} R u(k+j-1 \mid k)\right]
\end{aligned}
$$

subject to

$$
\begin{aligned}
& x(k \mid k)=x(k) \\
& x(k+j+1 \mid k)=A x(k+j \mid k)+B u(k+j \mid k) \\
& C x(k+j+1 \mid k) \leq D, \quad j=0,1, \ldots, N_{p}-1 \\
& S u(k \mid k) \leq T-S \Delta u^{*} \\
& S u(k+j \mid k) \leq T, \quad j=1, \ldots, N_{p}-1 .
\end{aligned}
$$

We denote the solution of $\mathcal{P}\left(x(k), \Delta u^{*}\right)$ as $\left\{u^{*}(k \mid k), u^{*}(k+\right.$ $\left.1 \mid k), \ldots, u^{*}\left(k+N_{p}-1 \mid k\right)\right\}$ and the corresponding predicted states as $\left\{x^{*}(k+1 \mid k), x^{*}(k+2 \mid k), \ldots, x^{*}\left(k+N_{p} \mid k\right)\right\}$.

Step 4: Implement the following control to the system (1):

$$
u(k)=u^{*}(k \mid k)+\Delta u^{*} .
$$

Proposition 1: If the optimization problems $\mathcal{P}_{\Delta}(w(k-1))$ and $\mathcal{P}\left(x(k), \Delta u^{*}\right)$ are both feasible, the state constraint satisfaction, i.e., $C x(k+1) \leq D$, can always be guaranteed if the control law (25) is applied to the linear system (1).
Proof: If the optimization problems $\mathcal{P}_{\Delta}(w(k-1))$ and $\mathcal{P}\left(x(k), \Delta u^{*}\right)$ are feasible, we have that the corresponding optimal solution satisfy the following constraints:

$$
\begin{aligned}
& C B \Delta u^{*} \leq-C w(k-1)-E \\
& C x^{*}(k+1 \mid k) \leq D \\
& S u^{*}(k \mid k) \leq T-S \Delta u^{*} .
\end{aligned}
$$

From (28), it follows that $S\left(u^{*}(k \mid k)+\Delta u^{*}\right) \leq T$, thus, the input constraints $S u(k) \leq T$ are satisfied.

Applying the control (25) and Assumption 1, the state $x(k+$ 1) is given by

$$
\begin{aligned}
x(k+1) & =A x(k)+B u(k)+w(k) \\
& =A x(k)+B\left(u^{*}(k \mid k)+\Delta u^{*}\right)+w(k) \\
& =x^{*}(k+1 \mid k)+B \Delta u^{*}+\hat{w}(k-1)+\varepsilon .
\end{aligned}
$$

Notice that inequalities (26) and (27) are already satisfied. Adding each side of (26) and (27), we have

$$
C B \Delta u^{*}+C x^{*}(k+1 \mid k) \leq-C \hat{w}(k-1)-E+D
$$

which, after rearranging the terms, leads to

$$
C x^{*}(k+1 \mid k)+C B \Delta u^{*}+C \hat{w}(k-1)+E \leq D .
$$

Since $E=\max (C \varepsilon), C \varepsilon \leq E$, then

$$
\begin{aligned}
C x(k+1) & =C x^{*}(k+1 \mid k)+C B \Delta u^{*}+C \hat{w}(k-1)+C \varepsilon \\
& \leq C x^{*}(k+1 \mid k)+C B \Delta u^{*}+C \hat{w}(k-1)+E \\
& \leq D .
\end{aligned}
$$

Therefore, the state constraints $C x(k+1) \leq D$ are satisfied. $\square$

Remark 2: The computational effort needed for the DC-MPC scheme is similar to the standard MPC scheme. In addition to the quadratic programming (QP) problem (which has the same structure as for the standard MPC) solved in Step 3, the DC-MPC scheme also solves an $n_{i}$-dimensional optimization problem in Step 2, where $n_{i}$ is the dimension of the control input. Compared with the QP problem in Step 3, which has a dimension of $N_{p} \times n_{i}$, such a low-dimension optimization problem does not involve much additional computational cost. As a result, the proposed DC-MPC scheme is much less computationally involved than the robust MPC algorithm discussed in [3], [4], and [10].

Remark 3: The minimization of the cost function in $\mathcal{P}_{\Delta}(\hat{w}(k-1))$ sets the DC-MPC scheme different from M-MPC scheme. In solving $\mathcal{P}_{\Delta}(\hat{w}(k-1))$, we attempt to make the response close to that without disturbance. Specifically, if $\|C B \Delta u+C \hat{w}(k-1)\|=0$ is satisfied, we have $x(k+1)=x^{*}(k+1 \mid k)+\varepsilon$, which means the states of the system with disturbances is made to be almost the same as the desired ones without disturbances.

Remark 4: The feasibility of $\mathcal{P}_{\Delta}(\hat{w}(k-1))$ is largely dependent on the properties of matrix $C B$, which indicates the control authority on the constrained states, and the magnitude of the disturbance. If the disturbance is too large or $C B$ is ill-conditioned, the required compensation might exceed the input limits, which results in infeasibility of $\mathcal{P}_{\Delta}(\hat{w}(k-1))$. Only if the required 


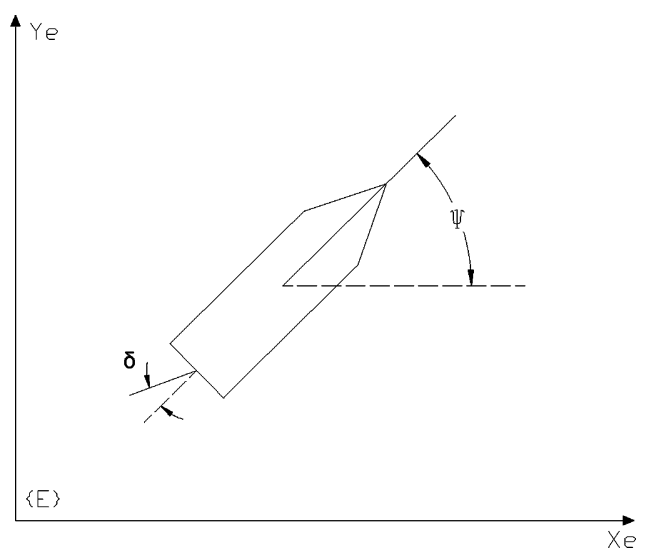

Fig. 1. Definition of ship heading and rudder angle.

compensation satisfies the input constraints, the feasibility of $\mathcal{P}_{\Delta}(\hat{w}(k-1))$ can be guaranteed. For the application of ship heading control, where the rudder action has enough control authority on the constrained state (yaw rate), the feasibility of $\mathcal{P}_{\Delta}(\hat{w}(k-1))$ can be guaranteed if wave disturbance is not extremely large.

Remark 5: The assumption of the feasibility of $\mathcal{P}\left(x(k), \Delta u^{*}\right)$ might not hold for some systems under certain conditions. Particularly, if one of the constrained states is the direct integral of other state, repeated feasibility cannot be guaranteed even if the state constraints are satisfied initially. For example, if there are position constraints for a dynamical positioning system, the initial position of the ship in the feasible region cannot guarantee the feasibility of $\mathcal{P}\left(x(k), \Delta u^{*}\right)$. Specifically, when the ship is on the boundary of the feasible region, and the ship speed is large and pointing outside the feasible region, $\mathcal{P}\left(x(k), \Delta u^{*}\right)$ might be infeasible. These problems are inherent to most of the MPC applications, and are not particular features of the proposed DC-MPC scheme. The problem is often dealt with by defining the invariant set and restraining the state within such a set.

\section{DC-MPC APPLICATION IN SHIP HEADING CONTROL}

\section{A. Introduction of Ship Heading Control}

Ship heading control, or the so-called course keeping, is the primary task of autopilots. The control objective of ship heading control is to make

$$
\psi \rightarrow \psi_{d}
$$

where $\psi$ is ship's actual heading angle, and $\psi_{d}$ is the desired ship heading angle, which is normally assumed to be constant [1]. Notice $\dot{\psi}=r$, where $r$ is the yaw rate.

For the ship heading control design, the Nomoto model is by far the most commonly employed one in the literature [2]. The Nomoto model considers 1 DOF ship dynamics, namely, the yaw rate $r$, and one control input, namely, the rudder angle $\delta$. The illustration of ship heading and rudder angle is shown in Fig. 1. Please note the other degrees of freedom of the ship have been neglected.
In this paper, the container ship S175 [1] is adopted as an example. For S175, the corresponding $1 \mathrm{DoF}$ linear dynamics in continuous time are given by

$$
\dot{x}=A_{c} x+B_{c} \delta+w
$$

where $x=[r, \psi]^{T}$ and

$$
A_{c}=\left[\begin{array}{cc}
-0.1068 & 0 \\
1 & 0
\end{array}\right] \quad B_{c}=\left[\begin{array}{c}
0.0028 \\
0
\end{array}\right] .
$$

Given a specific sampling time $T_{s}$, the plant (34) is easily transformed into its discrete-time version of (1). For this application, where $T_{s}=0.5 \mathrm{~s}$, the discrete-time system matrices are given by

$$
A=\left[\begin{array}{ll}
0.9480 & 0 \\
0.4869 & 1
\end{array}\right] \quad B=\left[\begin{array}{l}
0.0014 \\
0.0003
\end{array}\right] .
$$

Normally, the rudder saturates due to the physical limit. Furthermore, to avoid abrupt turns, which may induce undesirable ship motion, a yaw rate limit is considered in the control design. Therefore, the corresponding matrices $C, D, S$, and $T$ are given by

$$
\begin{aligned}
& C=\left[\begin{array}{cc}
1 & 0 \\
-1 & 0
\end{array}\right] \quad D=\left[\begin{array}{l}
0.006 \\
0.006
\end{array}\right] \\
& S=\left[\begin{array}{c}
1 \\
-1
\end{array}\right] \quad T=\left[\begin{array}{l}
35 \pi / 180 \\
35 \pi / 180
\end{array}\right] .
\end{aligned}
$$

For the ship control problem with yaw rate limit, the feasibility of $\mathcal{P}_{\Delta}(\hat{w}(k-1))$ is guaranteed if the disturbance is not too large because the rudder input has enough authority on yaw rate, and $C B=[0.0014,-0.0014]^{T}$ is well conditioned. For example, for a container ship S175 in a sea state 5 wave field, the maximum value of $C w$ is $[0.0005,-0.0005]^{T}$ with sampling time $T_{s}=0.5 \mathrm{~s}$. In this case, the maximum variation of the disturbance can be determined as $E=[0.00005,0.00005]^{T}$. Thus, the constraints (17) and (18) for $\mathcal{P}_{\Delta}(\hat{w}(k-1))$ are easily satisfied. The feasibility of $\mathcal{P}\left(x(k), \Delta u^{*}\right)$ can be guaranteed if the yaw rate constraints are satisfied initially, since the yaw rate is controlled directly by the input and is not the direct integral of other states.

To illustrate the capability of DC-MPC, it is first implemented on the linear model with sinusoidal and constant disturbances, and the performance is compared with those of the standard and M-MPC schemes. Then, the DC-MPC scheme is applied to the original nonlinear system in wave fields for the performance validation.

\section{B. Simulation Results: Linear Model With Constant and Sinusoidal Disturbances}

Two disturbances are considered in this case. One is sinusoidal $(0.001 \sin (0.08 t))$ and the other is constant $(-0.0015)$, which mimic the first-order and second-order wave disturbance effects [17]. In this particular study, the rudder constraints are $|\delta| \leq 35^{\circ}$ and the yaw rate constraints are $|r| \leq 0.006 \mathrm{rad} / \mathrm{s}$ $\left(0.34^{\circ} / \mathrm{s}\right)$.

The standard MPC scheme is first studied by simulations, with the results summarized in Fig. 2. In the simulations, $T_{s}=$ 

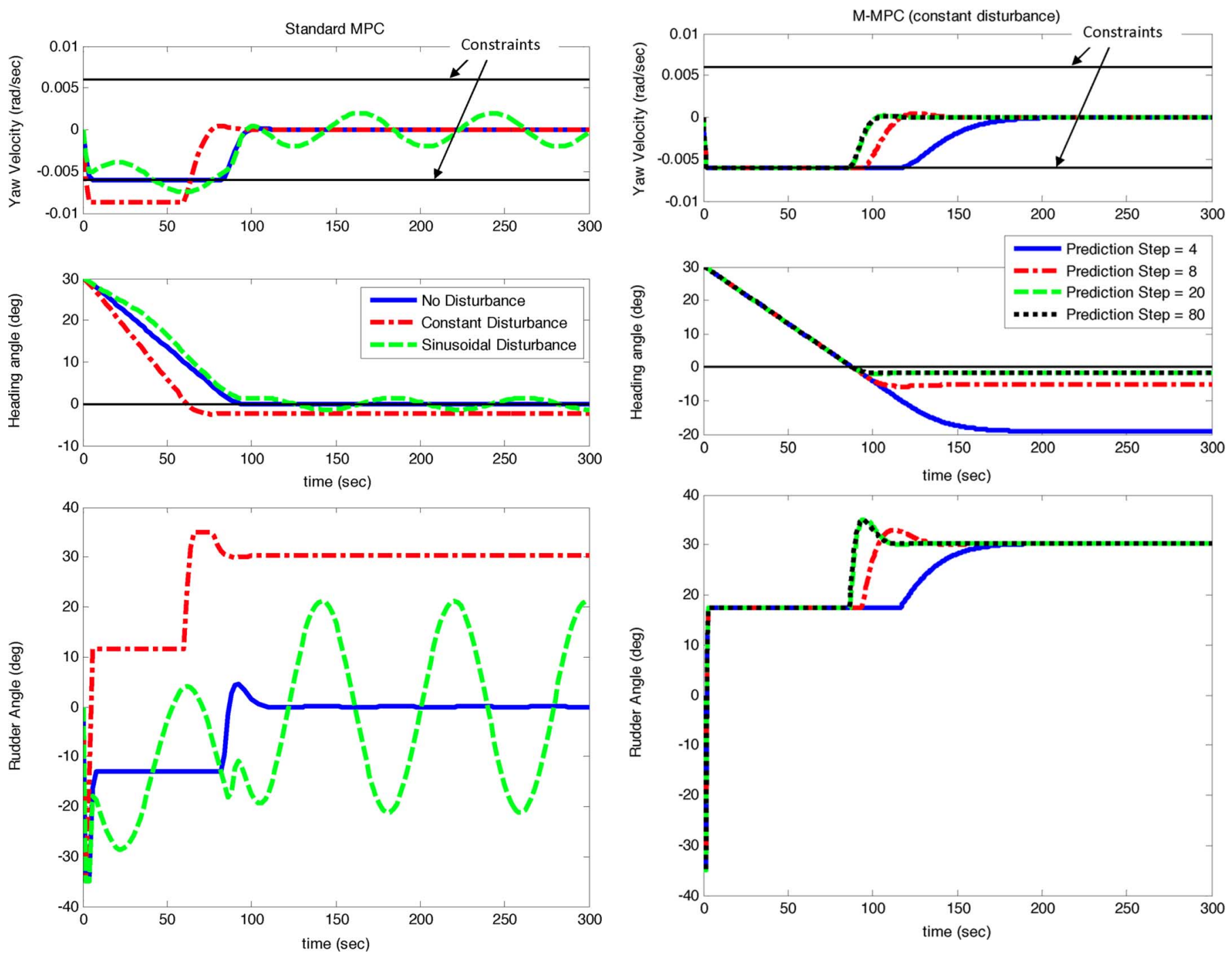

Fig. 2. Standard MPC ship heading controller simulations with and without disturbances.

$0.5 \mathrm{~s}, N_{p}=80, Q=\{1000,300\}$, and $R=1$. The parameters are chosen to achieve good performance in calm water. Fig. 2 shows that although the standard MPC scheme achieves good performance in calm water in terms of meeting constraints and achieving desired heading, the performance of the standard MPC in the presence of disturbances is not satisfactory. First, the yaw constraint violations are observed with both constant and sinusoidal disturbances. Second, a steady-state error exists in the constant disturbance case, while heading angle oscillations are observed with the sinusoidal disturbance.

Remark 6: In the simulation to obtain the results shown in Fig. 1, it occurred in many time steps that the optimization problem of the standard MPC has no solution for the given state constraints. At these time steps, the yaw rate constraints are temporarily removed to avoid the breakdown of the MPC controller so that the simulation can continue, and the hard constraint on the input is imposed on the rudder.

The M-MPC and DC-MPC are also implemented with different prediction horizons to study their performance with constant and sinusoidal disturbances. The simulations of the
Fig. 3. Simulations of the M-MPC ship heading controller with constant disturbances for different prediction horizons.

M-MPC are summarized in Figs. 3 and 4, while those of the DC-MPC are shown in Figs. 5 and 6, for constant and sinusoidal disturbances, respectively. In these simulations, the controller parameters are kept the same as the standard MPC.

As shown in Figs. 3 and 4, although the yaw constraints are successfully enforced by the M-MPC for all prediction horizons, the heading angle tracking performance is not satisfactory, particularly with those having short prediction horizons. Longer prediction horizon results in better performance for both constant and sinusoidal disturbances. However, the steady-state error cannot be completely eliminated.

Figs. 5 and 6 show that the DC-MPC has the capability to satisfy the state (yaw) constraints for all prediction horizons with both constant and sinusoidal disturbances. Furthermore, the DC-MPC scheme can eliminate the steady-state error with the constant disturbance for all prediction horizons. It also largely reduces the heading angle oscillations with the sinusoidal disturbance compared with the standard MPC and M-MPC cases. With longer prediction horizons, the DC-MPC scheme achieves better performance in terms of faster heading angle tracking and less heading angle oscillations. 

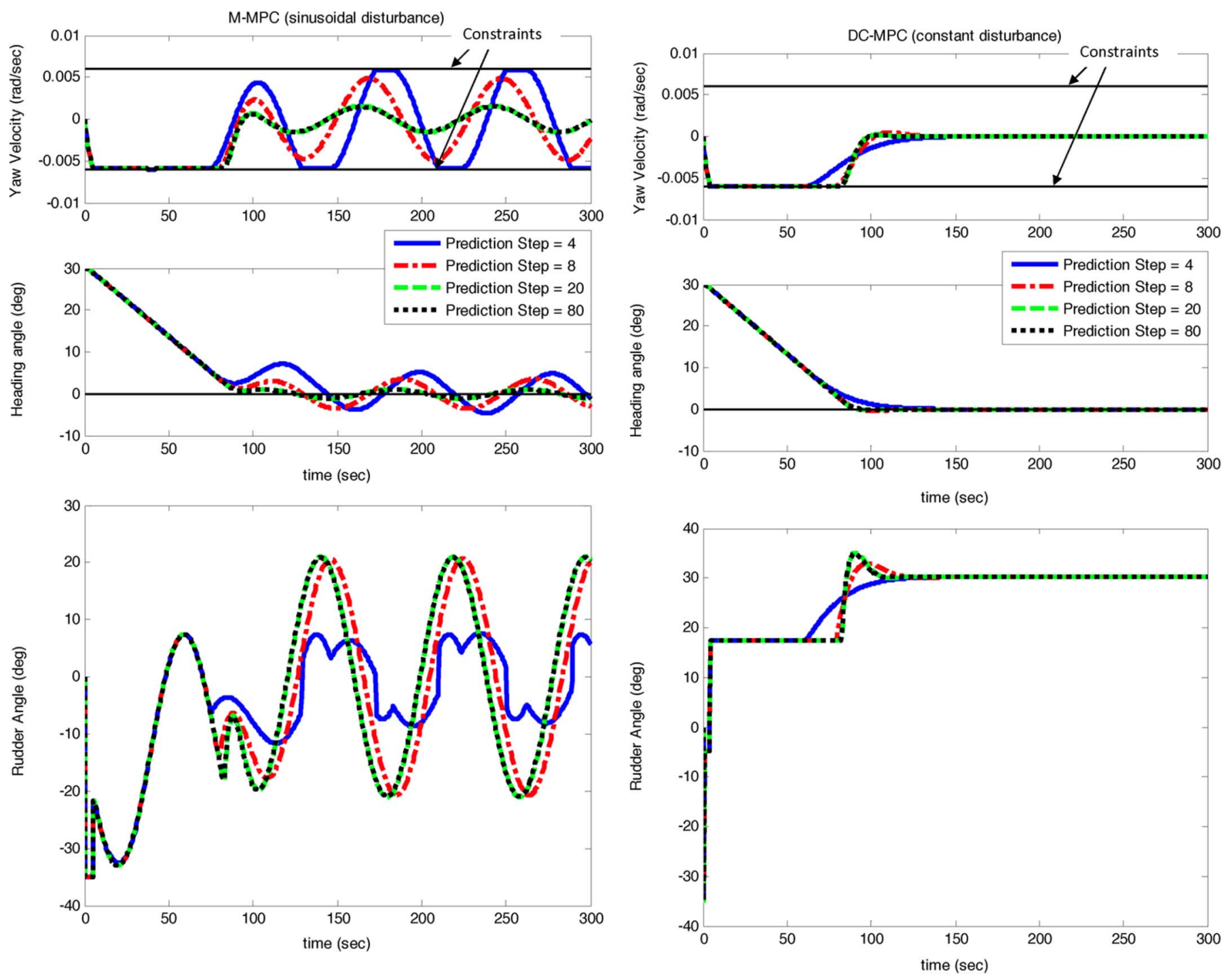

Fig. 4. Simulations of the M-MPC ship heading controller with sinusoidal disturbances for different prediction horizons.

For the case of -0.0015 constant disturbance in Fig. 5, the rudder angle to compensate the disturbance is about $30^{\circ}$. This result indicates that the maximum disturbance magnitude the system (with a maximum $35^{\circ}$ rudder angle) can handle to avoid state constraint violation is about 0.0016 .

The comparisons of the results of both M-MPC and DC-MPC schemes with a sinusoidal disturbance are summarized in Fig. 7. Fig. 7 shows that the DC-MPC has less heading angle oscillations than the M-MPC. The amplitude of heading angle oscillations with the DC-MPC is around $0.2^{\circ}$, while that with the M-MPC is around $0.9^{\circ}$. Furthermore, the capability of the DC-MPC scheme to track the system response without disturbances is illustrated (also see Figs. 5 and 6), which is discussed in Remark 3.

To quantitatively evaluate the controller performance, the comparison of performance indices for the DC-MPC and M-MPC under disturbances, both constant and sinusoidal, is summarized in Table I. It is shown from Table I that the DC-MPC scheme has better performance in terms of steady
Fig. 5. Simulations of the DC-MPC ship heading controller with constant disturbances for different prediction horizons.

state and accumulated errors for all simulation conditions considered.

The different approaches adopted by the M-MPC and DC-MPC lead to the performance differences. The M-MPC scheme minimizes the cost function based on the predictions of the nominal system (considering only the disturbance in one time step); thus, the mismatch of the nominal system and real system results in the steady-state error (constant disturbance) or state oscillations (sinusoidal disturbance). In contrast, the DC-MPC scheme is trying to track the desired no-disturbance performance (minimize the distance between the actual states and the predicted optimal states without disturbance), which results in steady-state error elimination and state oscillations reduction. The DC-MPC algorithm has the potential to be applied to other motion control problems with environmental disturbances, such as flight, automobile. and robotics controls, since in these,cases the system response without disturbances is always designed to be desirable. 

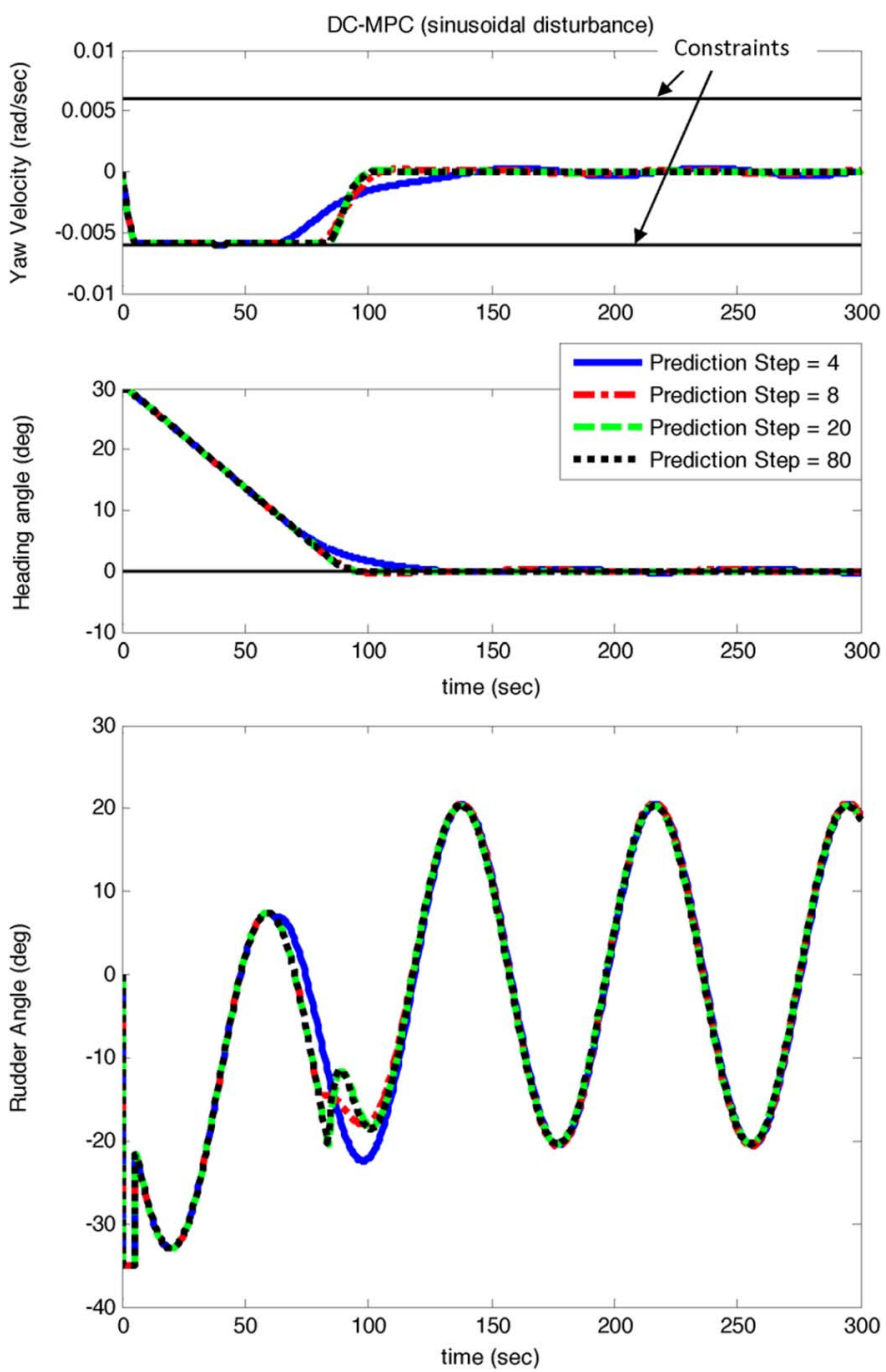

Fig. 6. Simulations of the DC-MPC ship heading controller with sinusoidal disturbances for different prediction horizons.

\section{Simulation Results: Nonlinear System With Wave Disturbances}

To further validate its performance, the DC-MPC scheme is evaluated and compared with M-MPC scheme on the numerical test-bed developed in [17]. The wave-induced yaw moment is predicted by linear seakeeping theory (first order) and empirical equation (second order). The simulation results, compared with the standard MPC without yaw constraints, are summarized in Fig. 8. In the simulations, sea state 5 is used, and the initial ship heading angle with respect to the wave heading angle is $0^{\circ}$. The same sampling time and predictive horizon are used, namely, $T_{s}=0.5 \mathrm{~s}$ and $N_{p}=80$. Fig. 8 shows that the DC-MPC scheme successfully enforces the yaw rate constraints. The initial course-changing speed for the DC-MPC is slower than the standard MPC without yaw constraints, while the final convergence speeds for both cases are similar. However, the yaw velocity constraint violations were observed in the M-MPC case. Furthermore, it can be observed from Fig. 8 that the yaw angle response of the DC-MPC is closer to the desired response (the solid black line in yaw angle subplot) than the M-MPC case. As discussed in Remark 2, the computational time for DC-MPC
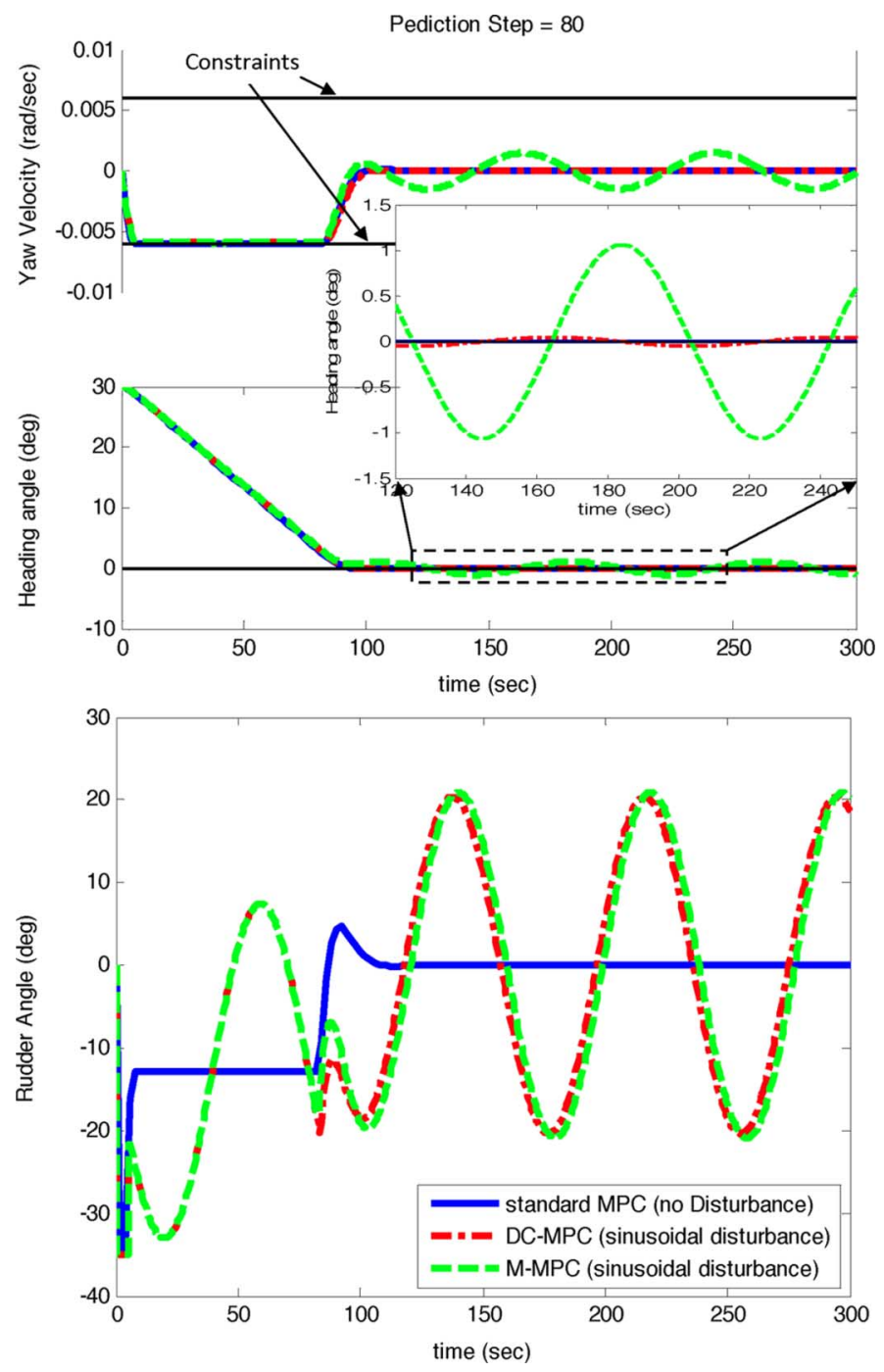

Fig. 7. Comparisons of the standard MPC (without disturbance), M-MPC (with disturbance), and DC-MPC (with disturbance) ship heading controller.

TABLE I

PERFORMANCE INDEX COMPARISONS OF DC-MPC AND M-MPC

\begin{tabular}{|c|r|r|r|}
\hline & $N_{p}$ & DC-MPC & M-MPC \\
\hline Steady State & 4 & 0 & 19.16 \\
\cline { 2 - 4 } Error [deg] & 8 & 0 & 5.31 \\
\cline { 2 - 4 }$($ Constant & 20 & 0 & 1.75 \\
\cline { 2 - 4 } Disturbance) & 80 & 0 & 1.65 \\
\hline \hline $\int_{0}^{t_{\text {final }}|\psi|} \mid d t$ & 4 & 1.4844 & 2.1042 \\
{$\left[\right.$ deg.sec $\left.\times 10^{3}\right]$} & 8 & 1.4239 & 1.8536 \\
\cline { 2 - 4 }$($ Sinusoidal & 20 & 1.4997 & 1.5535 \\
\cline { 2 - 4 } Disturbance) & 80 & 1.4987 & 1.5429 \\
\hline
\end{tabular}

and M-MPC schemes are very similar because the DC-MPC scheme just requires to solve an additional 1-D optimization problem in this case. Model mismatch is expected in the simulations because the linear MPC algorithm is adopted for the nonlinear ship control. However, the simulation results show that DC-MPC scheme has the robustness against such model mismatch, although the theoretical analysis is not given.

Fig. 8 shows that the DC-MPC can satisfy the yaw constraints in the course-changing stage. However, large rudder actions, for compensating the wave disturbance, are observed in the course- 

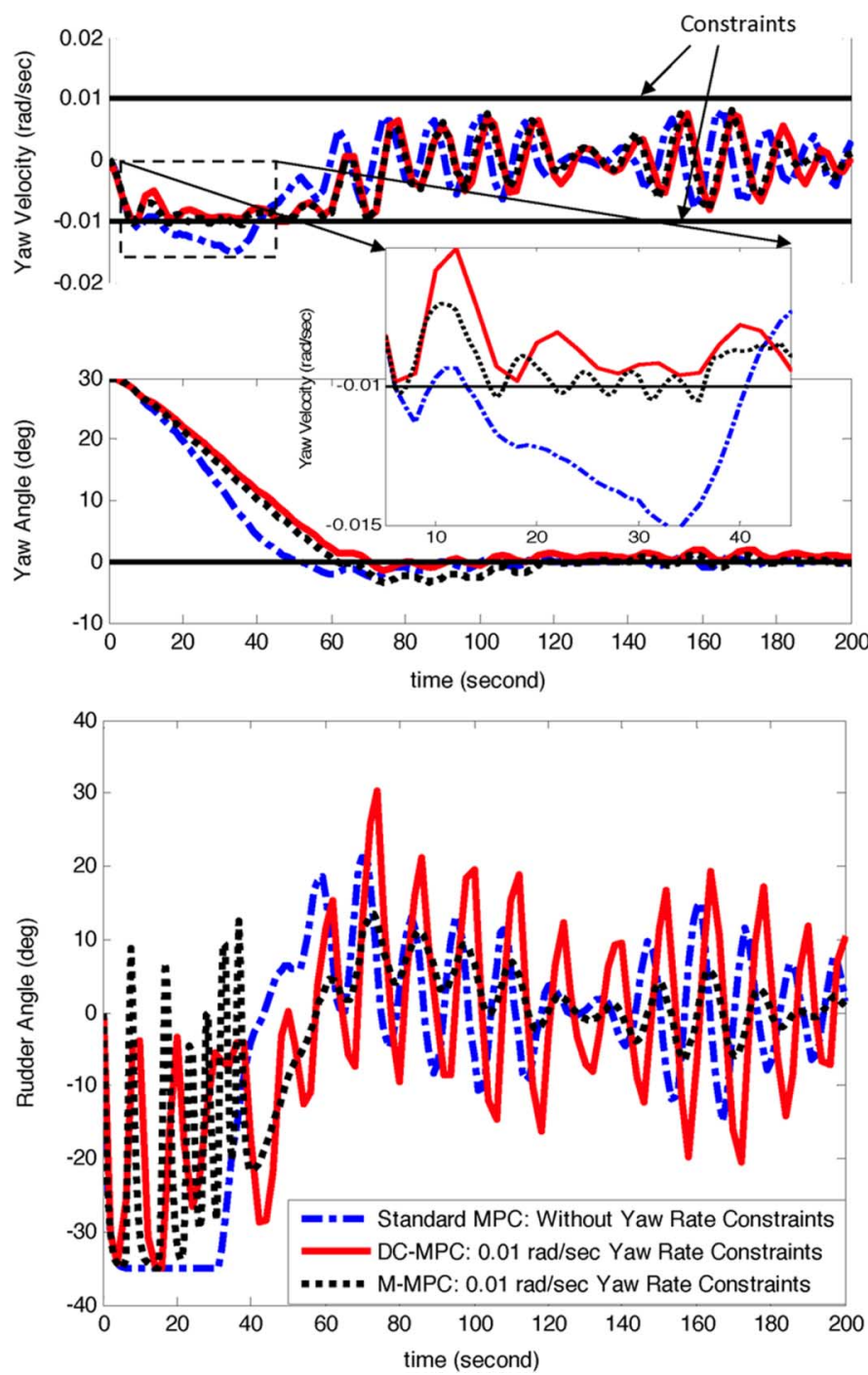

Fig. 8. Simulations of DC-MPC scheme and M-MPC applied to the nonlinear system in wave fields.

keeping stage. The rudder oscillations in the course-keeping stage cause wear and tear of the steering gear, and thus, need to be eliminated. Therefore, the DC-MPC should be modified to eliminate the rudder oscillations when the vessel has already approached the desired heading. In such circumstances, the yaw velocity constraint violation is normally no longer a consideration.

For this particular purpose, the disturbance compensating control action (calculated in Step 2) is set to zero when the heading error is less than $1^{\circ}$. The reason of choosing $1.0^{\circ}$ rather than smaller angle as a threshold is that the wave field is random, and often, a large wave can push the ship to deviate from the steady state more than $0.8^{\circ}$. If a very small degree threshold has been chosen, the algorithm will switch back and forth between DC-MPC and modified DC-MPC, which introduces additional rudder oscillations. Furthermore, the value of $R$ used in Step 3 (penalty on the rudder angle) is increased, and the additional penalty on rudder angle changing is introduced to avoid large rudder actions. This approach is similar to the gain scheduling adopted in [17]. In this case, $R=100$ and $\bar{R}=150$, where $\bar{R}$ is
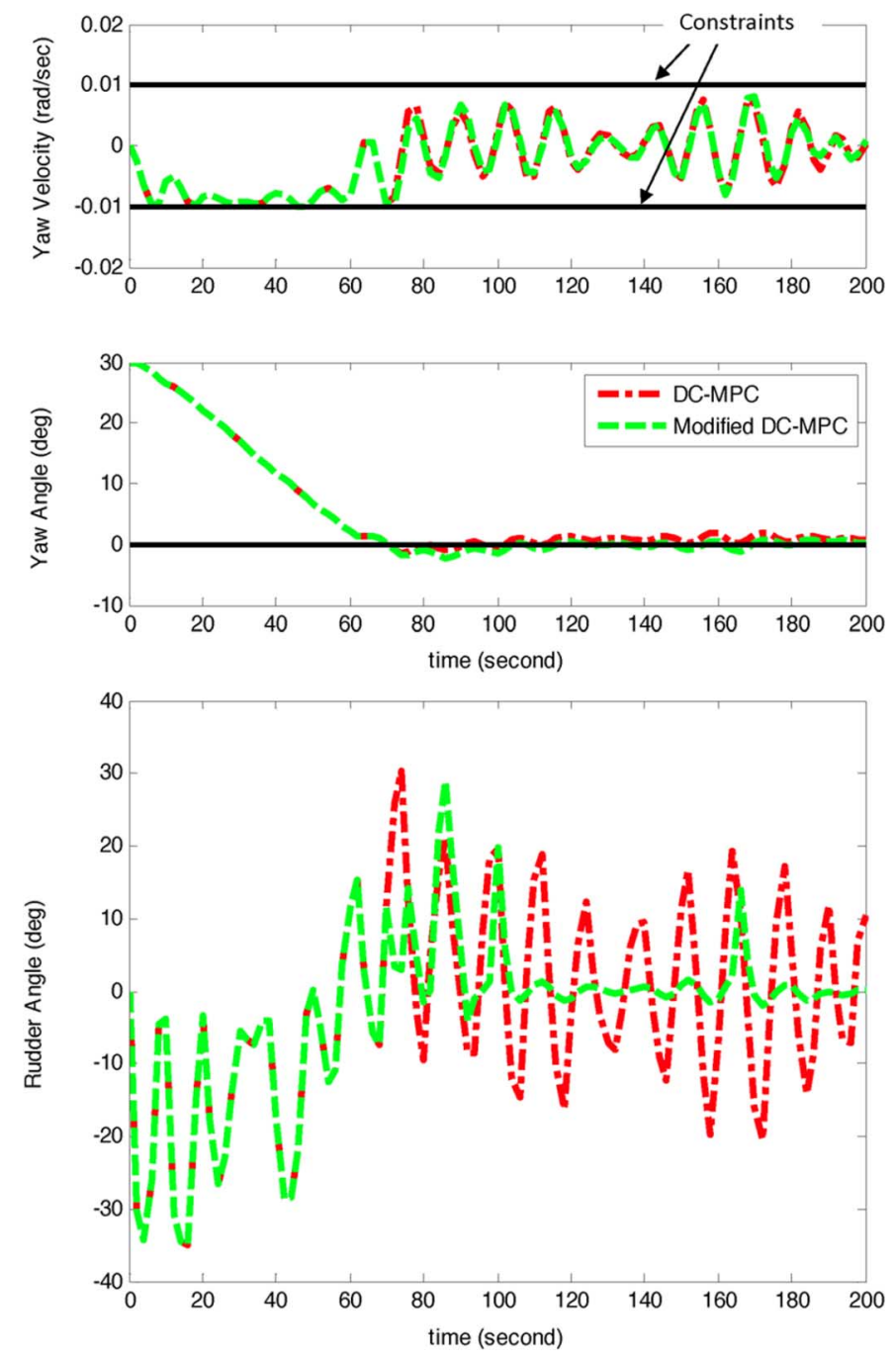

Fig. 9. Simulations of modified DC-MPC scheme applied to the nonlinear system in wave fields.

the penalty on the rudder angle changing (these two parameters are determined by extensive simulations). The comparison of simulations of DC-MPC and modified DC-MPC is summarized in Fig. 9. Fig. 9 shows that the modified DC-MPC not only satisfies the yaw rate constraints, but also eliminates the excessive rudder oscillations in the course-keeping stage. Please note that a wave filter or observer can be also employed to reduce the rudder oscillations. In such cases, the state constraints cannot be explicitly imposed.

\section{CONCLUSION}

In this paper, the DC-MPC scheme was developed to compensate for disturbances and to address infeasibility of the optimization problem associated with MPC formulation. A simple disturbance estimation and compensating method was first introduced and discussed. Then, the theoretical analysis was performed to show that DC-MPC can satisfy state constraints and achieve target performance under a given set of assumptions. The DC-MPC scheme was applied to ship heading control on a linear model, and compared with standard and M-MPC. The simulations show that the DC-MPC can 
eliminate the drawbacks of standard MPC, thereby satisfying the state constraints, eliminating the steady-state error, and reducing the state and control oscillations. The simulation results also show that better performance are achieved by the use of DC-MPC scheme over the M-MPC scheme. Furthermore, the performance of DC-MPC scheme was validated by simulations for the original nonlinear vessel model with nonlinear and time-varying wave disturbances. In fact, comparing the DC-MPC with a robust MPC in terms of conservativeness would be of interest as the latter does not explore the specific properties of the disturbance other than its robustness. We intend to explore this direction in our future work for more general class of systems.

\section{REFERENCES}

[1] T. Fossen, Guidance and Control of Ocean Vehicles. Hoboken, NJ:: Wiley, 1994

[2] T. Fossen, "Marine Control Systems," Marine Cybernetics, Trondheim, Norway, 2002.

[3] S. Qin and T. Badgwell, "A survey of industrial model predictive control technology," Control Eng. Practice, vol. 11, pp. 733-764, 2003.

[4] J. Rawlings and D. Mayne, Model Predictive Control Theory and Design. Madison, WI: Nob Hill Publishing, 2009.

[5] E. Gilbert, I. Kolmanovsky, and K. Tan, "Discrete-time reference governors and the nonlinear control of systems with state and control constraints," Int. J. Robust Nonlinear Control, vol. 5, pp. 487-504, 1995.
[6] A. Wahl and E. Gilles, "Track-keeping on waterways using model predictive control," in Proc. IFAC Conf. Control Appl. Marine Syst., 1998, pp. 149-154.

[7] T. Perez, C. Tzeng, and G. Goodwin, "Model predictive rudder roll stabilization control for ships," presented at the 5th IFAC Conf. Maneuvering Control Marine Craft, Aalborg, Denmark, 2000.

[8] Z. Li, J. Sun, and S. Oh, "Path following for marine surface vessels with rudder and roll constraints: An MPC approach," in Proc. Amer. Control Conf., 2009, pp. 3611-3616.

[9] P. Scokaert and J. Rawlings, "Feasibility issues in linear model predictive control," Amer. Inst. Chem. Eng. J., vol. 45, no. 8, pp. 1649-1659, 1999.

[10] D. Mayne, J. Rawlings, C. Rao, and P. Scokaert, "Constrained model predictive control: Stability and optimality," Automatica, vol. 36, pp. 789-814, 2000

[11] A. Zheng and M. Morari, "Robust stability of constrained model predictive control," in Proc. Amer. Control Conf., 1993, pp. 379-383.

[12] P. Scokaert and D. Mayne, "Min-max feedback model predictive control for constrained linear systems," IEEE Trans. Autom. Control, vol. 43, no. 8, pp. 1136-1142, Aug. 1998.

[13] L. Chisci, J. Rossiter, and G. Zappa, "Systems with persistent disturbances: Predictive control with restrictive constraints," Automatica, vol. 37, pp. 1019-1028, 2001.

[14] Y. Lee and B. Kouvaritakis, "Robust receding control for systems with uncertain dynamics and input saturation," Automatica, vol. 36, pp. $1497-1504,2000$.

[15] D. Mayne, M. Seron, and S. Rakovic, "Robust model predictive control of constrained linear systems with bounded disturbances," Automatica, vol. 41, pp. 1136-1142, 2005.

[16] R. Ghaemi, J. Sun, and I. Kolmanovsky, "Computationally efficient model predictive control with explicit disturbance mitigation and constraint enforcement," in Proc. 45th IEEE Conf. Decision Control, 2006 pp. 4842-4847.

[17] Z. Li, J. Sun, and R. Beck, "Evaluation and modification of a robust path following controller for marine surface vessels in wave fields," $J$. Ship Res., vol. 54, pp. 141-147, 2010. 